\title{
Consumo de drogas ilícitas como fator de risco para traumatismo dentário em adolescentes
}

\author{
Illicit drugs use as risk factor of dental trauma among \\ adolescents
}

\author{
Haroldo Neves de Paiva ${ }^{1}$, Paula Cristina Pelli Paiva ${ }^{1}$, Carlos José de Paula Silva ${ }^{1}$, \\ Joel Alves Lamounier ${ }^{2}$, Efigênia Ferreira e Ferreira ${ }^{3}$, Patrícia Maria Zarzar ${ }^{4}$
}

\begin{abstract}
Resumo
Traumatismo dentário e consumo de drogas ilícitas podem comprometer gravemente a saúde dos adolescentes e são considerados sérios problemas de saúde pública. Objetivo: Investigar a associação do traumatismo dentário com o uso de drogas ilícitas e condição socioeconômica entre adolescentes de 12 anos de idade. Métodos: Estudo transversal envolvendo 633 adolescentes de escolas públicas e privadas da cidade de Diamantina, no Estado de Minas Gerais. Os dados foram coletados por meio de exame clínico e questionários. Traumatismo dentário foi avaliado pela classificação de Andreasen, e consumo de drogas ilícitas, pelo instrumento ASSIST (Teste para Triagem do Envolvimento com Álcool, Cigarro e Outras Substâncias). As associações foram testadas pelo teste do qui-quadrado e teste Exato de Fisher $(\mathrm{p}<0,05)$. Resultados: A presença do traumatismo dentário foi observada em 176 adolescentes (29,9\%). A prevalência reportada do uso de maconha foi de 1,5\% (9/588), de cocaína, 0,3\% (2/588), e de inalantes, 1,7\% (10/588). Traumatismo dentário foi mais prevalente entre adolescentes do sexo masculino $(p=0,010)$ que tinham usado maconha $(p=0,024)$ na vida. Conclusão: A associação observada entre o uso de drogas ilícitas e o sexo masculino com o traumatismo dentário sugere a necessidade de adoção de políticas voltadas para o controle efetivo dessas condições, principalmente em idade precoce.
\end{abstract}

Palavras-chave: traumatismos dentários; saúde bucal; adolescentes; drogas ilícitas.

\begin{abstract}
The dental trauma and consumption of illicit drugs can seriously jeopardize adolescents' health and are considered serious public health problems. Objective: To investigate the association between dental trauma and the use of illicit drugs and socioeconomic status among adolescents 12 years of age. Methods: Cross-sectional study involving 633 adolescents enrolled in public and private schools of the city Diamantina-MG. Data were collected through self-administered questionnaires and clinical examination. The dental trauma was assessed by Andreasen classification and consumption of illicit drugs was investigated using the ASSIST instrument (Involvement Screening Test for with Alcohol, Cigarette and Other Substances). Associations were tested using the Chi-square test and Fisher's Exact test $(p<0.05)$. Results: The presence of dental trauma was observed in 176 adolescents $(29.9 \%)$. The reported prevalence of marijuana use was $1.5 \%(9 / 588), 0.3 \%$ cocaine (2/588) and inhalants $1.7 \%(10 / 588)$. Dental trauma was more prevalent among adolescent males $(p=0.010)$ who had used marijuana $(p=0.024)$ in life. Conclusion: The observed association between illicit drug use and male with dental trauma suggests the need to adopt policies for the effective control of these conditions especially at an early age.
\end{abstract}

Keywords: tooth injuries; oral health; adolescent; street drugs.

'Programa de Pós-graduação em Odontologia, Faculdade de Ciências Biológicas e da Saúde, Universidade Federal dos Vales do Jequitinhonha e Mucuri (UFVJM) - Diamantina (MG), Brasil.

${ }^{2}$ Departamento de Medicina, Universidade Federal de São João Del Rei (UFSJ) - São João del-Rei (MG), Brasil.

${ }_{3}^{3}$ Programa de Pós-graduação em Saúde Coletiva, Faculdade de Odontologia, Universidade Federal de Minas Gerais (UFMG) - Belo Horizonte (MG), Brasil. ${ }_{4}^{4}$ Programa de Pós-graduação em Odontopediatria e Ortodontia, Faculdade de Odontologia, Universidade Federal de Minas Gerais (UFMG) - Belo Horizonte (MG), Brasil.

Trabalho realizado nas escolas públicas e privadas da cidade de Diamantina - Diamantina (MG), Brasil.

Endereço para correspondência: Haroldo Neves de Paiva - Rua da Glória, 187 - Centro - CEP: 39100-000 - Diamantina (MG), Brasil - Email: hnevesp@gmail.com Fonte de financiamento: Bolsa de doutorado CAPES.

Conflito de interesses: nada a declarar. 


\section{INTRODUÇÃO}

Adolescência é uma etapa essencial na vida, caracterizada, principalmente, pelo esforço dos jovens em consolidar sua independência utilizando, muitas vezes, mecanismos que englobam a rejeição dos valores sociais convencionais, religiosos e familiares estabelecidos pelos pais e educadores ${ }^{1}$. Assim, não é surpreendente que a adolescência seja reconhecida como uma idade de vulnerabilidade ${ }^{2}$, durante a qual ocorrem as experiências com substâncias psicoativas legais e ilegais ${ }^{3,4}$.

O uso de drogas por adolescentes constitui um importante problema social e de saúde pública ${ }^{5}$, pois apresenta alta prevalência e início cada vez mais precoce ${ }^{6}$. A Organização Mundial de Saúde (OMS) estima que $0,6 \%$ da população tenha problemas associados ao uso de drogas ilícitas ${ }^{6-8}$. Entre os transtornos associados ao uso de substâncias, destacam-se perda de memória, baixo desempenho escolar ou no trabalho, distúrbios físicos e psicológicos, dependência na vida adulta, suicídio, acidentes ou violência culminando em trauma, como o traumatismo facial e dentário ${ }^{5-12}$.

O traumatismo dentário (TD) pode ser definido como qualquer injúria de natureza térmica, química ou física que afete o dente e representa, dentre as alterações bucais, um dos mais sérios problemas de saúde pública em crianças e adolescentes ${ }^{13}$. Consideram-se lesões traumáticas dentárias desde uma fratura em esmalte até a perda definitiva do elemento dentário ${ }^{14}$. Na dentição permanente, a maioria dos estudos cita uma prevalência média de aproximadamente $20 \%$, com valores variando de 4,1 a $58,6 \%{ }^{15}$.

A literatura é carente de trabalhos que avaliem a associação do TD e o uso de drogas ilícitas por adolescentes. Em busca realizada nas bases de dados, apenas dois estudos avaliaram essa associação, apresentando resultados controversos ${ }^{10,16}$. Dessa forma, o desenvolvimento de estudos epidemiológicos que investiguem a associação do consumo de drogas por escolares com TD e fatores associados é de fundamental importância a fim de que $o$ atendimento a pacientes traumatizados inclua não somente o tratamento odontológico, mas também a implantação, nas escolas, de programas educacionais que visem à redução do consumo de drogas. A necessidade de se desenvolver meios de identificar a associação entre o uso de substâncias e o TD é condição sine qua non para combater de forma eficaz esse consumo.

O objetivo do presente estudo foi avaliar a associação do TD com sexo, uso de drogas ilícitas e condição socioeconômica em adolescentes de 12 anos de idade da cidade de Diamantina, no Estado de Minas Gerais, bem como avaliar a prevalência do consumo dessas drogas.

\section{MÉTODOS}

\section{Desenho de estudo e participantes}

Foi realizado um estudo transversal de base populacional na área urbana de Diamantina, Sudeste brasileiro, durante os meses de fevereiro a abril de 2013. O município contava com aproximadamente 46.372 habitantes e um índice de desenvolvimento humano (IDH) de 0,716. O Vale do Jequitinhonha, onde a cidade de Diamantina encontra-se inserida, faz parte da Agência de Desenvolvimento do Nordeste (ADENE). Em 2012, Diamantina possuía 7.474 estudantes distribuídos na zona rural e urbana, dos quais 477 matriculados na rede particular e 6.997 na rede pública estadual e municipal de ensino. $\mathrm{O}$ estudo foi do tipo censo e envolveu todos os escolares de 12 anos. A adoção dessa idade justificou-se por ser a recomendada pelo OMS para levantamento de saúde bucal e também por ter o intuito de investigar a precocidade com que os adolescentes estão iniciando a utilização de tais substâncias psicoativas. Foram convidados a participar 633 escolares de 13 escolas públicas e privadas da zona urbana. As seguintes estimativas foram obtidas para a amostra: prevalência de TD em grupos expostos e não expostos, as razões de prevalência (RP), o nível de confiança de $95 \%$ e um poder de teste de $80 \%$.

\section{Estudo-piloto}

Com o objetivo de adequar a metodologia, um estudo-piloto foi realizado no ano anterior em amostra de conveniência com 101 estudantes de escolas públicas e privadas, que não foram incluídos na amostra do estudo principal. Após a realização do estudo-piloto, verificou-se que não seriam necessárias modificações na metodologia.

\section{Instrumentos e variáveis}

A variável dependente do estudo - a presença de TD - foi investigada segundo os critérios diagnósticos propostos por Andreasen ${ }^{14}$, que incluem dente, tecido periodontal, mucosa bucal e injúrias ao osso de suporte. Foi eleita esta classificação por ser de fácil memorização e de ordenação lógica do TD: (1) fratura de esmalte; (2) fratura de esmalte e dentina; (3) fratura de esmalte e dentina com exposição pulpar; (4) luxação; (5) intrusão; (6) extrusão; (7) luxação lateral; (8) avulsão. A equipe constou de um examinador (P.C.P.P), previamente treinado e calibrado (intra-examiner Kappa $=0,79$; inter-examiner Kappa=0,85), e de um anotador. Realizou-se a coleta de dados na própria escola em horário e dia previamente agendados. A iluminação e o tipo de mobília para exame foram padronizados, com utilização de cadeira escolar para os adolescentes assentarem-se e iluminação natural e artificial (Petzl Zoom headlamp; Petzl America, Clearfield, UT, USA). Adotaram-se os princípios de biossegurança por meio de equipamentos de proteção individual, bem como instrumentais autoclavados. Os dentes foram limpos e secos 
com gaze, enquanto a coroa dental, examinada em relação à perda de substância dentária, intrusão, extrusão, luxação lateral, avulsão ou presença de restaurações devido ao TD. As variáveis independentes foram avaliadas por intermédio de aplicação de questionário investigando o uso de drogas ilícitas na vida. As variáveis demográficas e socioeconômicas foram coletadas por formulário, enviado aos pais/responsáveis com o Termo de Consentimento Livre e Esclarecido (TCLE).

Avaliou-se o uso ilícito de drogas pela primeira pergunta do questionário ASSIST (Teste para Triagem do Envolvimento com Álcool, Cigarro e Outras Substâncias), com opção de resposta sim ou não. Esse questionário foi desenvolvido pela OMS visando aos cuidados primários de saúde, nos quais o uso de substâncias nocivas pode passar despercebido. Muitos profissionais de saúde podem diagnosticar a dependência nos pacientes, mas não serem capazes de identificar o uso perigoso ou nocivo. O ASSIST contém oito questões sobre o uso de nove classes de substâncias psicoativas (tabaco, álcool, maconha, cocaína, estimulantes, sedativos, inalantes, alucinógenos e opiáceos) e foi validado no Brasil ${ }^{17}$. As questões abordam a frequência de uso na vida e nos últimos três meses. No Brasil, o instrumento ASSIST foi aplicado em adolescentes de 15 a 19 anos na cidade de Belo Horizonte, Minas Gerais ${ }^{16}$, e em adolescentes de 14 a 19 anos na cidade de Diamantina ${ }^{10}$.

A renda familiar foi um dos indicadores socioeconômicos adotados. Ela baseou-se na somatória dos salários recebidos pelos membros economicamente ativos residentes com os adolescentes, dividindo-se pelo salário mínimo vigente no período de realização do estudo, e foi dicotomizada pela mediana em valor $<3$ e $\geq 3$ salários mínimos. O número de anos estudados pela mãe foi também outro indicador investigado, e seus valores foram dicotomizados pela mediana em até sete anos de estudo e oito anos ou mais. De acordo com o Instituto Brasileiro de Geografia e Estatística (IBGE) ${ }^{18}$, a média de idade de estudo da população brasileira é 7,4 anos, portanto o ponto de corte de sete anos escolhido para avaliar a escolaridade materna coincide com o término do ensino fundamental. Os valores foram dicotomizados em 0 e 1 para baixa e alta classe socioeconômica, respectivamente.

Os estudantes receberam os questionários codificados. A autoaplicação destes foi realizada em sala de aula, na ausência do professor. O pesquisador leu em voz alta todos os itens para que todos respondessem simultaneamente, evitando a troca de informações e também resguardando de preconceitos os que não possuíam proficiência na leitura ${ }^{19}$. O sigilo e a privacidade foram garantidos.

\section{Considerações éticas}

O estudo foi aprovado pelo Comitê de Ética em Pesquisa da Universidade Federal de Minas Gerais (COEP-317/11). As autorizações da Superintendência Regional de Ensino e das escolas participantes foram devidamente obtidas e o TCLE também foi assinado pelos participantes e seus pais/responsáveis. A confidencialidade quanto à identificação foi garantida e mantida a todos os participantes. Todos os adolescentes portadores de TD com indicação para reabilitação foram encaminhados à Prefeitura Municipal de Diamantina para a devida assistência odontológica.

\section{Análise estatística}

A análise dos dados foi realizada com o programa SPSS Statistical Package for the Social Sciences (SPSS for Windows, version 19.0, SPSS Inc, Chicago, IL, USA). Realizou-se análise descritiva com medidas de tendência central para a caracterização da amostra. Análise univariada foi utilizada para verificar a associação entre a variável dependente - TD - e as variáveis independentes, adotando o teste do qui-quadrado de Pearson e o teste Exato de Fisher $(p<0,05)$.

\section{RESULTADOS}

A amostra final foi constituída por 588 escolares. A perda de $7,11 \%(n=45)$ foi composta de estudantes não autorizados pelos pais/responsáveis ou que não quiseram participar do estudo. Do total de escolares, 48,6\% $(n=286)$ eram do sexo masculino. A Tabela 1 caracteriza a amostra segundo as variáveis socioeconômicas.

O TD nos incisivos permanentes foi observado em 176 escolares $(29,9 \%)$ e foi maior no sexo masculino $(34,2 \%)$ do que no feminino (24,6\%), com diferença estatisticamente significativa $(\mathrm{p}<0,010)$ (Tabela 2).

Quanto à prevalência do uso de substâncias psicoativas, $7,1 \%(n=42)$ dos escolares usaram derivados do tabaco, $46,8 \%$ $(n=278)$, bebidas alcoólicas, $1,5 \%(n=9)$, maconha, $0,7 \%(n=4)$, cocaína, $1,7 \%(n=10)$, inalantes, e $0,2 \%(n=1)$, alucinógenos (Tabela 3). Adolescentes que relataram ter usado maconha tiveram uma prevalência de TD maior na análise univariada,

Tabela 1. Características demográficas e socioeconômicas da amostra de 588 adolescentes de 12 anos de idade da cidade de Diamantina, em Minas Gerais

\begin{tabular}{lcc}
\multicolumn{1}{c}{ Variável } & n & \% \\
Sexo & & \\
$\quad$ Feminino & 302 & 51,4 \\
$\quad$ Masculino & 286 & 48,6 \\
Tipo de escola & & \\
$\quad$ Particular & 46 & 7,8 \\
$\quad$ Pública & 542 & 92,2 \\
Escolaridade materna & & \\
0-7 anos & 212 & 36,1 \\
8 ou mais anos & 376 & 63,9 \\
Renda familiar & & \\
1/2 a 3 salários mínimos & 443 & 75,3 \\
$\quad$ 3 salários mínimos & 145 & 24,7 \\
\hline
\end{tabular}


Tabela 2. Distribuição de 588 adolescentes com 12 anos de idade de acordo com a presença de traumatismo dentário e as variáveis demográficas e socioeconômicas na cidade de Diamantina, em Minas Gerais

\begin{tabular}{|c|c|c|c|c|}
\hline \multirow[b]{2}{*}{$\begin{array}{c}\text { Variáveis } \\
\text { independentes }\end{array}$} & \multicolumn{2}{|c|}{ Traumatismo dentário } & \multirow[b]{2}{*}{$p^{*}$} & \multirow[b]{2}{*}{ OR IC95\% } \\
\hline & $\begin{array}{l}\text { Presença de } \\
\text { traumatismo }\end{array}$ & $\begin{array}{l}\text { Ausência de } \\
\text { traumatismo }\end{array}$ & & \\
\hline Sexo & (n) (\%) & (n) $(\%)$ & & \\
\hline Feminino & $76(25,2)$ & $226(74,8)$ & $0,010^{*}$ & $1,59(1,12-2,28)$ \\
\hline Masculino & $100(35,0)$ & $186(65,0)$ & & \\
\hline \multicolumn{5}{|l|}{ Renda familiar } \\
\hline <3 salários mínimos & $135(30,5)$ & $307(69,5)$ & $0,605^{\star}$ & $0,89(0,59-1,35)$ \\
\hline 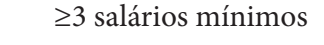 & $41(28,3)$ & $104(71,7)$ & & \\
\hline \multicolumn{5}{|l|}{ Escolaridade materna } \\
\hline $0-7$ anos & $69(32,9)$ & $141(67,1)$ & $0,237^{\star}$ & $1,24(0,86-1,79)$ \\
\hline 8 ou mais anos & $106(28,2)$ & $270(71,8)$ & & \\
\hline
\end{tabular}

*Teste do qui-quadrado de Pearson

Tabela 3. Distribuição de 588 adolescentes com 12 anos de idade de acordo com a presença de traumatismo dentário e as variáveis de consumo de drogas ilícitas na cidade de Diamantina, em Minas Gerais

\begin{tabular}{|c|c|c|c|c|}
\hline \multirow{2}{*}{$\begin{array}{c}\text { Variáveis } \\
\text { independentes }\end{array}$} & \multicolumn{2}{|c|}{ Traumatismo dentário } & \multirow[b]{2}{*}{$p^{*}$} & \multirow[b]{2}{*}{ OR IC95\% } \\
\hline & $\begin{array}{l}\text { Presença de } \\
\text { traumatismo }\end{array}$ & $\begin{array}{l}\text { Ausência de } \\
\text { traumatismo }\end{array}$ & & \\
\hline \multicolumn{5}{|l|}{ Usou derivados de tabaco } \\
\hline Não & $164(30,0)$ & $382(70,0)$ & $0,842^{*}$ & $0,93(0,45-1,86)$ \\
\hline Sim & $12(28,6)$ & $30(71,4)$ & & \\
\hline \multicolumn{5}{|l|}{ Usou bebidas alcoólicas } \\
\hline Não & $85(27,2)$ & $228(72,8)$ & $0,117^{\star}$ & $1,33(0,93-1,89)$ \\
\hline Sim & $91(33,1)$ & $184(66,9)$ & & \\
\hline \multicolumn{5}{|l|}{ Usou maconha } \\
\hline Não & $170(29,4)$ & $409(70,6)$ & $0,024^{* *}$ & $4,81(1,19-10,46)$ \\
\hline Sim & $6(66,7)$ & $3(33,3)$ & & \\
\hline \multicolumn{5}{|l|}{ Usou cocaína } \\
\hline Não & $173(29,6)$ & $411(70,4)$ & $0,082^{* *}$ & $7,12(0,73-68,99)$ \\
\hline Sim & $3(75,0)$ & $1(25,0)$ & & \\
\hline \multicolumn{5}{|l|}{ Usou inalante } \\
\hline Não & $173(29,9)$ & $405(70,1)$ & $0,617^{\star *}$ & $1,03(0,25-3,92)$ \\
\hline Sim & $3(30,0)$ & $7(70,0)$ & & \\
\hline \multicolumn{5}{|l|}{ Usou alucinógenos } \\
\hline Não & $176(30,0)$ & $411(70,0)$ & $0,701^{\star *}$ & $0,70(0,66-0,73)$ \\
\hline Sim & $0(0)$ & $1(100)$ & & \\
\hline
\end{tabular}

${ }^{*}$ Teste do qui-quadrado de Pearson;

${ }^{*}$ Teste Exato de Fisher

e essa associação foi estatisticamente significante $(\mathrm{p}=0,024$; $\mathrm{OR}=4,812$; IC95\%=1,190-10,412) (Tabela 3). Portanto, os adolescentes que relataram uso de maconha tiveram 4,8 vezes mais chances de pertencer ao grupo acometido por TD.

\section{DISCUSSÃO}

Das alterações bucais, o TD representa um dos mais sérios problemas de saúde bucal em crianças e adolescentes devido à alta prevalência e ao alto impacto psicossocial ${ }^{20}$. No presente estudo, 29,9\% dos adolescentes apresentaram pelo menos um dente traumatizado. Por causa da baixa perda ocorrida, a amostra estudada pode ser considerada como representativa. Há uma grande variação entre os valores de prevalência observados em diversas regiões do mundo ${ }^{21}$, refletindo uma diversidade metodológica, seja nos critérios diagnósticos adotados, faixa etária da população estudada, diferenças culturais ou comportamentais. Este estudo apresentou valores semelhantes a outros trabalhos nacionais ${ }^{22,23}$, cuja metodologia e idade dos adolescentes foram similares.

Existe consenso na literatura a respeito da associação do TD com os fatores clínicos predisponentes ${ }^{15,10,21,24,25}$, porém Glendor ${ }^{21}$ sugeriu que fatores comportamentais, socioeconômicos e ambientais tenham provavelmente maior importância para o TD do que fatores clínicos. 
Nas buscas realizadas nas bases de dados PubMed, Medline, Embase, SciELO no período de 2005 a 2015, foram encontradas apenas dois estudos relacionando TD ao uso de substâncias psicoativas em idades entre 14 a $19 \operatorname{anos}^{10,16}$. A adoção da idade de 12 anos neste caso justificou-se no intuito de investigar a precocidade com que os adolescentes estão iniciando a utilização de tais substâncias e na escassez de estudos relacionando-as ao TD.

O sexo masculino apresentou maior risco de ocorrência de TD, bem como para consumo de substâncias psicoativas ${ }^{10}$. Essa relação foi estatisticamente significativa, corroborando a maioria dos estudos nos quais o sexo masculino apresentou até o dobro de lesões traumáticas ${ }^{10,16,22}$.

Ao considerar associação do TD com a condição socioeconômica, não foi observada nenhuma associação estatística. Uma revisão crítica da literatura sobre os indicadores socioeconômicos concluiu que, na maioria dos estudos, também não foi encontrada essa associação ${ }^{26}$.

A maconha é a droga ilícita mais consumida em todo do mundo ${ }^{27}$. Assim, diante das consequências decorrentes de seu $\mathrm{uso}^{5,6}$ e corroborando a literatura, os adolescentes que relataram o consumo de maconha apresentaram uma associação estatisticamente significante com a presença de TD, ou seja, até 4,8 mais chances de pertencerem ao grupo com lesões traumáticas dentárias. Embora a variável uso na vida de drogas não seja computada no escore geral do instrumento ASSIST, ela foi utilizada por ser uma informação que denuncia a vulnerabilidade social dos adolescentes em relação às drogas ${ }^{10,28,29}$, principalmente na idade estudada, e a precocidade com que esse uso vem ocorrendo. Segundo $\mathrm{Abbey}^{30}$, quanto mais precoce o início do consumo de drogas, maior o risco de surgirem consequências graves ${ }^{28,29}$ devido às intensas transformações que ocorrem na adolescência, devendo os profissionais que lidam com essa temática estarem atentos a essa questão.

O código penal brasileiro considera como infração o uso de drogas ilícitas, passível de punição tanto para o usuário como para o seu responsável legal. Ressalta também que esse uso deve ser abordado como um problema de saúde pública $\left(\text { Lei } \mathrm{n}^{\circ} 11.343 / 2006\right)^{31}$. O consumo de drogas ilícitas pelos adolescentes neste estudo mostrou-se preocupante, uma vez que se trata de uma população vulnerável, devido às intensas transformações que ocorrem na fase de transição entre a infância e a vida adulta, que se encontra incluída no sistema educacional.

Embora não tenha sido observada associação entre o TD e o consumo de álcool na vida, este se apresentou como a substância mais consumida. A crença de que a bebida alcoólica não é droga contribui para o estímulo e incentivo ao seu consumo não só durante a adolescência, mas em todas as faixas etárias ${ }^{32}$. Assim, torna-se necessário reforçar a importância da orientação sobre os efeitos prejudiciais dessas substâncias no início da idade escolar, o que poderia prevenir a experimentação precoce e o uso abusivo na adolescência e nos adultos jovens.

Estudos realizados em diferentes contextos corroboram os resultados aqui apresentados, alertando para o consumo frequente de álcool, tabaco e uso de outras drogas entre adolescentes ${ }^{3,4,11,33,34}$, porém tais comparações devem ser estabelecidas com cautela em virtude de características culturais inerentes a cada população estudada.

A menor prevalência de consumo das outras drogas investigadas talvez possa ser explicada pela faixa etária estudada ou também pela omissão dos escolares sobre suas experiências passadas. No entanto, não podemos esquecer que este estudo foi realizado no ambiente escolar, tendo esses adolescentes mais acesso a informações e, provavelmente, melhores condições de vida do que os evadidos do sistema educacional.

Algumas limitações metodológicas devem ser consideradas. Por se tratar de um estudo transversal, uma relação de causalidade não pode ser determinada. Além disso, nossa abordagem não incluiu os indivíduos que foram excluídos ou que evadiram da escola.

\section{CONCLUSÃO}

A associação observada entre o TD, uso de drogas ilícitas e o sexo masculino sugere a necessidade de adoção de políticas voltadas para o controle efetivo dessas condições. Assim, ressalta-se a importância da detecção e intervenção precoce com relação ao uso de drogas por escolares, possibilitando o planejamento e o desenvolvimento de ações preventivas de acordo com seus respectivos contextos.

\section{REFERÊNCIAS}

1. World Health Organization. Improving Health trough schools: national and international strategies [Internet]. 2015 [citado em 2015 jul 10]. Disponível em: http://www.who.int/features/factfiles/adolescent_health/ facts/en/index6.html

2. Senna SRCM, Dessen MA. Contribuições das teorias do desenvolvimento humano para a concepção contemporânea da adolescência. Psic Teor e Pesq. 2012;28(1):101-8. http://dx.doi.org/10.1590/S0102-37722012000100013.

3. Andrade AG, Duarte PC, Barroso LP, Nishimura R, Alberghini DG, Oliveira LG. Use of alcohol and other drugs among Brazilian college students: effects

of gender and age. Rev Bras Psiquiatr. 2012;34(3):294-305. PMid:23429775. http://dx.doi.org/10.1016/j.rbp.2012.02.002.

4. Schenker M, Minayo MCS. Fatores de risco e de proteção para o uso de drogas na adolescência. Cien Saude Colet. 2005;10(3):707-17. http://dx.doi. org/10.1590/S1413-81232005000300027.

5. Hall W, Degenhardt L. High potency cannabis: a risk factor for dependence, poor psychosocial outcomes, and psychosis. BMJ. 2015;4(350):h1205. PMid:25739398. http://dx.doi.org/10.1136/bmj.h1205. 
6. Hoch E, Bonnetn U, Thomasius R, Ganzer F, Havemann-Reinecke U, Preuss UW. Risks associated with the non-medicinal use of cannabis. Dtsch Arztebl Int. 2015;112(16):271-8. PMID: 25939318. http://dx.doi. org/10.3238/arztebl.2015.0271.

7. World Health Organization. Management of Substance Abuse. The WHO ASSIST Project [Internet]. Geneva: WHO; 2008 [citado 2013 abr 15]. Disponível em: www.who.int/substance_abuse/activities/ehealth/en/

8. Degenhardt L, Hall W, Warner-Smith M, Lynskey M. Illicit drug use. In: World Health Organization. Comparative quantifi cation of health risks: global and regional burden of disease attributable to selected major risk factors. Geneva: WHO; 2004. 1175 p.

9. Room R, Babor T, Rehm J. Alcohol and public health. Lancet. 2005;365(9458):51930. PMid:15705462. http://dx.doi.org/10.1016/S0140-6736(05)70276-2.

10. Oliveira Filho PM, Jorge KO, Paiva PC, Ferreira EF, Ramos-Jorge ML, Zarzar PM. The prevalence of dental trauma and its association with illicit drug use among adolescents. Dent Traumatol. 2013;30(2):122-7. PMid:23841700. http://dx.doi.org/10.1111/edt.12059.

11. Vieira LP, Santana VTP, Suchara EA. Caracterização de tentativas de suicídios por substâncias exógenas. Cad Saude Colet. 2015;23(2):118-23. http://dx.doi.org/10.1590/1414-462X201500010074.

12. Loch MR, Bortoletto MSS, Souza RKT, Mesas AE. Simultaneidade de comportamentos de risco para a saúde e fatores associados em estudo de base populacional. Cad Saude Colet. 2015;23(2):180-7. http://dx.doi. org/10.1590/1414-462X201500020045.

13. Traebert J, Almeida ICS, Garghetti C, Marcenes WM. Prevalência, necessidade de tratamento e fatores predisponentes. Cad Saude Publica. 2004;20(2):40310. PMid:15073619. http://dx.doi.org/10.1590/S0102-311X2004000200007.

14. Andreasen JO, Andreasen FM, Andersson L. Textbook and color atlas of traumatic injuries to the teeth. 4th ed. Copenhagen: Munskgaard International Publishers, Wiley-Blackwell; 2007.

15. Anderson L. Epidemiology of traumatic dental injuries. Pediatr Dent. 2013;35(2):102-5. PMid:23635975.

16. Jorge KO, Oliveira Fo PM, Ferreira EF, Oliveira AC, Vale MP, Zarzar PM. Prevalence and association of dental injuries with socioeconomic conditions and alcohol/drug use in adolescents between 15 and 19 years of age. Dent Traumatol. 2012;28(2):136-41. PMid:21989043. http://dx.doi. org/10.1111/j.1600-9657.2011.01056.x.

17. Henrique IFS, Micheli D, Lacerda RB, Formigoni MLOS. Validation of the Brazilian version of alcohol, smoking and substance Involvement Screening Test (ASSIST). Rev Assoc Med Bras. 2004;50(2):199-206. PMid:15286871. http://dx.doi.org/10.1590/S0104-42302004000200039.

18. Instituto Brasileiro de Geografia e Estatística. Censo demografico 2010 dados dos distritos MG [Internet]. 2014 [citado em 2014 jul 28]. Disponível em: http://www.bnb.gov.br/content/aplicacao/prodetur/downloads/docs/ mg_7_2_inventar_oferta_turistica_informac_basica_distrito_100708.pdf

19. Narvai PC, Antunes JLF, Moysés SJ, Frazão P, Peres MA, Peres KG. Scientifi c validity of epidemiological knowledge based on data from the Brazilian Oral Health Survey (SB Brazil 2003). Cad Saude Publica. 2010;26(4):64770. PMid:20512200.

20. Glendor U. Aetiology and risk factors related to traumatic dental injuries--a review of the literature. Dent Traumatol. 2009;25(1):19-31. PMid:19208007. http://dx.doi.org/10.1111/j.1600-9657.2008.00694.x.
21. Glendor U. Epidemiology of traumatic dental injuries--a 12 year review of the literature. Dent Traumatol. 2008;24(6):603-11. PMid:19021651. http:// dx.doi.org/10.1111/j.1600-9657.2008.00696.x.

22. Traebert J, Marcon KB, Lacerda JT. Prevalence of traumatic dental injuries and associated factors in schoolchildren of Palhoça, Santa Catarina State. Cien Saude Colet. 2010;15(15 Suppl 1):1849-55. PMid:20640348. http:// dx.doi.org/10.1590/S1413-81232010000700098.

23. Damé-Teixeira N, Alves LS, Susin C, Maltz M. Traumatic dental injury among 12-year-old South Brazilian schoolchildren: prevalence, severity, and risk indicators. Dent Traumatol. 2013;29(1):52-8. PMid:22453035. http://dx.doi.org/10.1111/j.1600-9657.2012.01124.x.

24. Bauss O, Freitag S, Röhling J, Rahman A. Influence of overjet and lip coverage on the prevalence and severity of incisor trauma. J Orofac Orthop. 2008;69(6):402-10. PMid:19169637. http://dx.doi.org/10.1007/s00056-008$8805-1$.

25. Nguyen QV, Bezemer PD, Habets L, Prahl-Andersen B. A systematic review of the relationship between overjet size and traumatic dental injuries. Eur J Orthod. 1999;21(5):503-15. PMid:10565091. http://dx.doi.org/10.1093/ ejo/21.5.503.

26. Bendo CB, Scarpelli AC, Vale MP, Zarzar PMA. Correlation between socioeconomic indicators and traumatic dental injuries: a qualitative critical literature review. Dent Traumatol. 2009;25(4):420-5. PMid:19519857. http:// dx.doi.org/10.1111/j.1600-9657.2009.00803.x.

27. Pabst A, Kraus L, Matos EG, Piontek D. Substanzkonsum und substanzbezogene Störungen in Deutschland im Jahr 2012. Sucht. 2013;59(6):321-31. http:// dx.doi.org/10.1024/0939-5911.a000275.

28. Faler CS, Câmara SG, Aerts DRGC, Alves GG, Béria JU. Family psychosocial characteristics, tobacco, alcohol, and other drug use, and teenage pregnancy. Cad Saude Publica. 2013;29(8):1654-63. PMid:24005930. http://dx.doi. org/10.1590/S0102-311X2013001200017.

29. Moura YG, Sanchez ZM, Opaleye ES, Neiva-Silva L, Koller SH, Noto AR. Drug use among street children and adolescents: what helps? Cad Saude Publica. 2012;28(7):1371-80. PMid:22729267. http://dx.doi.org/10.1590/ S0102-311X2012000700015.

30. Abbey S. Alcohol-related sexual assault: a common problem among college students. J Stud Alcohol. 2002;(14):118-28. PMid:12022717. http://dx.doi. org/10.15288/jsas.2002.s14.118.

31. DireitoNet. Lei de Drogas (Lei no 11.343/06) I [Internet]. 2015 [citado em 2015 jun 10]. Disponível em: http://www.direitonet.com.br/testes/exibir/65/ Lei-de-Drogas-Lei-no-11343-06-I?cta_src=artigo_exibir

32. Teevale T, Robinson E, Duffy S, Utter J, Nosa V, Clark T, et al. Binge drinking and alcohol-related behaviours amongst Pacific youth: a national survey of secondary school students. N Z Med J. 2012;30(125):60-70. PMid:22472713.

33. Vieira PC, Aerts DRGC, Freddo SL, Bittencourt A, Monteiro L. Uso de álcool, tabaco e outras drogas por adolescentes escolares em município do Sul do Brasil. Cad Saude Publica. 2008;24(11):2487-98. PMid:19009129. http://dx.doi.org/10.1590/S0102-311X2008001100004.

34. Malbergier A, Cardoso LRD, Amaral RA. Uso de substâncias na adolescência e problemas familiares. Cad Saude Publica. 2012;28(4):678-88. PMid:22488313. http://dx.doi.org/10.1590/S0102-311X2012000400007.

Recebido em: Mar. 30, 2016 Aprovado em: Set. 11, 2016 